\title{
McClelland-Type Upper Bounds for Graph Energy
}

\author{
Igor Milovanović $^{a, *}$, Emina Milovanovic ${ }^{a}$ \\ Şerife Burcu Bozkurt Altindag $\breve{g}^{b}$, Marjan Matejić ${ }^{a}$ \\ ${ }^{a}$ Faculty of Electronic Engineering, University of Niš, NǏs, Serbia \\ ${ }^{b}$ Yenikent Kardelen Konutlar, Seluklu, Konya, Turkey \\ igor@elfak.ni.ac.rs, ema@elfak.ni.ac.rs, \\ srf_burcu_bozkurt@hotmail.com, marjan.matejic@elfak.ni.ac.rs
}

(Received February 23, 2022)

\begin{abstract}
Let $\mathrm{G}$ be a simple graph of order $n$, without isolated vertices. An important spectrum-based graph invariant is the graph energy. In this paper, we obtain several new upper bounds of the McClelland type on graph energy and characterize graphs for which these bounds are best possible. The bounds represent generalization and improvement of some known results from the literature.
\end{abstract}

\section{Introduction}

Let $G=(V, E), V=\left\{v_{1}, v_{2}, \ldots, v_{n}\right\}$, be a simple connected graph with $n=|V|$ vertices, $m=|E|$ edges, with vertex degree sequence $d_{1} \geq d_{2} \geq$ $\cdots \geq d_{n}, d_{i}=d\left(v_{i}\right)$. The greatest, the second greatest, the smallest and the second smallest vertex degrees with be, respectively, denoted as $\Delta=$ $d_{1}, \Delta_{2}=d_{2}, \delta=d_{n}$, and $\delta_{2}=d_{n-1}$. Denote by $D=\operatorname{diag}\left(d_{1}, d_{2}, \ldots, d_{n}\right)$ the diagonal matrix of vertex degrees. Let $A=\left(a_{i j}\right)$, be the $(0,1)$ adjacency matrix of $G$. The eigenvalues of matrix $A, \lambda_{1} \geq \lambda_{2} \geq \cdots \geq \lambda_{n}$, are the (ordinary) eigenvalues of $G$.

${ }^{*}$ Corresponding author. 
One of the most studied spectrum-based invariant in graph theory is the graph (ordinary) energy defined in [10]. It is calculated as

$$
E(G)=\sum_{i=1}^{n}\left|\lambda_{i}\right| .
$$

More on energy of graphs and matrices one can find in monographs $[11,16]$ and papers $[25,26]$.

The sum of the $\alpha$-th powers of the degrees of a graph $G$

$$
{ }^{0} R_{\alpha}(G)=\sum_{i=1}^{n} d_{i}^{\alpha},
$$

is known as general zeroth-order Randić index [13]. It is also met under names general first Zagreb index [17] and variable first Zagreb index [20] (see also $[2,3])$. Here we are interested in the following special cases of ${ }^{0} R_{\alpha}(G)$ :

- The inverse degree or modified total adjacency index, $I D(G)=$ ${ }^{0} R_{-1}(G)[7,27]$.

- The zeroth-order connectivity index or zeroth-order Randić index, ${ }^{0} R(G)={ }^{0} R_{-1 / 2}(G)[14]$.

- The first Zagreb index, $M_{1}(G)={ }^{0} R_{2}(G)[12]$.

- The forgotten topological index, $F(G)={ }^{0} R_{3}(G)[9]$.

\section{Preliminaries}

In this section we list several bounds on $E(G)$ reported in the literature that are of interest for the present paper. Also, we recall some analytical inequalities that will be used in proofs of theorems.

Lemma 1. [19] Let $G$ be a graph with $n$ vertices and $m$ edges. Then

$$
E(G) \leq \sqrt{2 m n}
$$


Equality holds if and only if $G \cong \overline{K_{n}}$, or $G \cong \frac{n}{2} K_{2}$, for even $n$.

Lemma 2. [21] Let $G$ be a graph with $n \geq 2$ vertices and $m$ edges. Then

$$
E(G) \leq \sqrt{\Delta}+\sqrt{(n-1)(2 m-\Delta)}
$$

Equality holds if and only if $G \cong \overline{K_{n}}$, or $G \cong \frac{n}{2} K_{2}$, for even $n$.

The above inequality was proved in [1] also.

Lemma 3. [8] Let $G$ be a graph with $n \geq 3$ vertices and $m$ edges. Then

$$
E(G) \leq \sqrt{\Delta}+\sqrt{\delta}+\sqrt{(n-2)(2 m-\Delta-\delta)} .
$$

Equality holds if and only if $G \cong \overline{K_{n}}$, or $G \cong \frac{n}{2} K_{2}$, for even $n$.

Lemma 4. [5] Let $G$ be a graph with $n$ vertices and $m$ edges. Then

$$
E(G) \leq \sum_{i=1}^{n} \sqrt{d_{i}} .
$$

Equality holds if and only if $G \cong \overline{K_{n}}$, or $G \cong t \cdot K_{2} \cup(n-2 t) K_{1}, 1 \leq t \leq \frac{n}{2}$.

Remark. The inequality (4) is the fundamental one from which a number of upper bounds for $E(G)$ can be obtained. For example, in [8] it was proven that inequalities (2) and (3) can be obtained from (4). Also, it is not difficult do observe that the simplest proof of (1) can be derived using (4). Namely, since

$$
\sum_{i=1}^{n} \sqrt{d_{i}} \leq \sqrt{2 m n},
$$

from the above and (4), the inequality (1) directly follows. Inspired by this fact, based on (4) we will prove the inequalities that generalize (1), (2) and (3).

The following inequalities for the real number sequences will be used in the proofs of the results in the next section.

Lemma 5. [15] Let $a=\left(a_{i}\right), i=1,2, \ldots, n$, be a sequence of positive real 
numbers. Then

$$
\left(\sum_{i=1}^{n} \sqrt{a_{i}}\right)^{2} \leq(n-1) \sum_{i=1}^{n} a_{i}+n\left(\prod_{i=1}^{n} a_{i}\right)^{1 / n} .
$$

Equality holds if and only if $a_{1}=a_{2}=\cdots=a_{n}$.

Lemma 6. [24] Let $=\left(p_{i}\right), i=1,2, \ldots, n$ be a sequence of non-negative real numbers and $a=\left(a_{i}\right), i=1,2, \ldots, n$, a sequence of positive real numbers. Then, for any real $r \leq 0$ or $r \geq 1$, holds

$$
\left(\sum_{i=1}^{n} p_{i}\right)^{r-1} \sum_{i=1}^{n} p_{i} a_{i}^{r} \geq\left(\sum_{i=1}^{n} p_{i} a_{i}\right)^{r} .
$$

When $0 \leq r \leq 1$, the opposite inequality holds. Equality holds if and only either $r=0$, or $r=1$, or $a_{1}=a_{2}=\cdots=a_{n}$, or $p_{1}=p_{2}=\cdots=p_{t}=0$ and $a_{t+1}=\cdots=a_{n}$, or $p_{t+1}=\cdots=p_{n}=0$ and $a_{1}=\cdots=a_{t}$, for some $t, 1 \leq t \leq n-1$.

\section{Main results}

In the next theorem we establish an upper bound for the $E(G)$ in terms of parameters $n, m, \Delta, \Delta_{2}, \delta$ and $\delta_{2}$.

Theorem 1. Let $G$ be a graph of order $n \geq 4$ and size $m$ without isolated vertices. Then we have

$$
E(G) \leq \sqrt{\Delta}+\sqrt{\delta}+\sqrt{(n-2)(2 m-\Delta-\delta)-\frac{n-2}{2}\left(\sqrt{\Delta_{2}}-\sqrt{\delta_{2}}\right)^{2}} .
$$

Equality holds if and only if $G \cong \frac{n}{2} K_{2}$, where $n$ is even.

Proof. Having in mind the Lagrage's identity (see for example [23]), the 
following inequalities are valid

$$
\begin{gathered}
(n-2) \sum_{i=2}^{n-1} d_{i}-\left(\sum_{i=2}^{n-1} \sqrt{d_{i}}\right)^{2}=\sum_{2 \leq i<j \leq n-1}\left(\sqrt{d_{i}}-\sqrt{d_{j}}\right)^{2} \geq \\
\geq\left(\sqrt{\Delta_{2}}-\sqrt{\delta_{2}}\right)^{2}+\sum_{i=3}^{n-2}\left(\left(\sqrt{\Delta_{2}}-\sqrt{d_{i}}\right)^{2}+\left(\sqrt{d_{i}}-\sqrt{\delta_{2}}\right)^{2}\right) \geq \\
\geq\left(\sqrt{\Delta_{2}}-\sqrt{\delta_{2}}\right)^{2}+\frac{1}{2} \sum_{i=3}^{n-2}\left(\sqrt{\Delta_{2}}-\sqrt{\delta_{2}}\right)^{2}=\frac{n-2}{2}\left(\sqrt{\Delta_{2}}-\sqrt{\delta_{2}}\right)^{2} .
\end{gathered}
$$

From the above we have that

$$
\left(\sum_{i=1}^{n} \sqrt{d_{i}}-\sqrt{\Delta}-\sqrt{\delta}\right)^{2} \leq(n-2)\left(\sum_{i=1}^{n} d_{i}-\Delta-\delta\right)-\frac{n-2}{2}\left(\sqrt{\Delta_{2}}-\sqrt{\delta_{2}}\right)^{2},
$$

that is

$$
\sum_{i=1}^{n} \sqrt{d_{i}} \leq \sqrt{\Delta}+\sqrt{\delta}+\sqrt{(n-2)(2 m-\Delta-\delta)-\frac{n-2}{2}\left(\sqrt{\Delta_{2}}-\sqrt{\delta_{2}}\right)^{2}} .
$$

From the above and inequality (4), the inequality (7) immediately follows.

Equality in (8) holds if and only if $d_{i} \in\{\Delta, \delta\}$, for every $i, 2 \leq i \leq n-1$. Since graph $G$ has no isolated vertices, equality in (4) holds if and only if $G \cong \frac{n}{2} K_{2}$, for even $n$. This implies that equality in (7) holds if and only if $G \cong \frac{n}{2} K_{2}$, for even $n$.

Remark. Since $\left(\sqrt{\Delta_{2}}-\sqrt{\delta_{2}}\right)^{2} \geq 0$, we have that

$$
\begin{aligned}
E(G) & \leq \sqrt{\Delta}+\sqrt{\delta}+\sqrt{(n-2)(2 m-\Delta-\delta)-\frac{n-2}{2}\left(\sqrt{\Delta_{2}}-\sqrt{\delta_{2}}\right)^{2}} \leq \\
& \leq \sqrt{\Delta}+\sqrt{\delta}+\sqrt{(n-2)(2 m-\Delta-\delta)} .
\end{aligned}
$$

This means that inequality (7) is stronger than (3).

Proofs of the next theorems are analogous to that of Theorem 1, hence omitted.

Theorem 2. Let $G$ be a graph of order $n \geq 3$ and size $m$ without isolated 
vertices. Then we have

$$
E(G) \leq \sqrt{\Delta}+\sqrt{(n-1)(2 m-\Delta)-\frac{n-1}{2}\left(\sqrt{\Delta_{2}}-\sqrt{\delta}\right)^{2}} .
$$

Equality holds if and only if $G \cong \frac{n}{2} K_{2}$, for even $n$.

Remark. Since $\left(\sqrt{\Delta_{2}}-\sqrt{\delta}\right)^{2} \geq 0$, we have that

$$
\begin{aligned}
E(G) & \leq \sqrt{\Delta}+\sqrt{(n-1)(2 m-\Delta)-\frac{n-1}{2}\left(\sqrt{\Delta_{2}}-\sqrt{\delta}\right)^{2}} \leq \\
& \leq \sqrt{\Delta}+\sqrt{(n-1)(2 m-\Delta)} .
\end{aligned}
$$

This means that inequality (9) is stronger than (2).

Theorem 3. Let $G$ be a graph of order $n \geq 2$ and size $m$ without isolated vertices. Then we have

$$
E(G) \leq \sqrt{2 m n-\frac{n}{2}(\sqrt{\Delta}-\sqrt{\delta})^{2}} .
$$

Equality holds if and only if $G \cong \frac{n}{2} K_{2}$, for even $n$.

Remark. Since $(\sqrt{\Delta}-\sqrt{\delta})^{2} \geq 0$ we have that

$$
E(G) \leq \sqrt{2 m n-\frac{n}{2}(\sqrt{\Delta}-\sqrt{\delta})^{2}} \leq \sqrt{2 m n} .
$$

This means that (10) is stronger than (1).

In the next theorem we determine an upper bound for $E(G)$ in terms of number of vertices, number of edges and maximum and minimum vertex degrees.

Theorem 4. Let $G$ be a graph of order $n \geq 3$ and size $m$ without isolated vertices. Then we have

$$
E(G) \leq \sqrt{\Delta}+\sqrt{\delta}+\sqrt{(n-3)(2 m-\Delta-\delta)+(n-2)\left(\frac{\operatorname{det} D}{\Delta \delta}\right)^{1 /(n-2)}} .
$$

Equality holds if and only if $G \cong \frac{n}{2} K_{2}$, for even $n$. 
Proof. The inequality (5) can be considered in the form

$$
\left(\sum_{i=2}^{n-1} \sqrt{a_{i}}\right)^{2} \leq(n-3) \sum_{i=2}^{n-1} a_{i}+(n-2)\left(\prod_{i=2}^{n-1} a_{i}\right)^{1 /(n-2)} .
$$

For $a_{i}=d_{i}, i=2,3, \ldots, n-1$, the above inequality becomes

$$
\left(\sum_{i=2}^{n-1} \sqrt{d_{i}}\right)^{2} \leq(n-3) \sum_{i=2}^{n-1} d_{i}+(n-2)\left(\prod_{i=2}^{n-1} d_{i}\right)^{1 /(n-2)}
$$

that is

$\sum_{i=1}^{n} \sqrt{d_{i}}-\sqrt{\Delta}-\sqrt{\delta} \leq \sqrt{(n-3)\left(\sum_{i=1}^{n} d_{i}-\Delta-\delta\right)+(n-2)\left(\frac{\prod_{i=1}^{n} d_{i}}{\Delta \delta}\right)^{1 /(n-2)}}$

from which we obtain

$$
\sum_{i=1}^{n} \sqrt{d_{i}} \leq \sqrt{\Delta}+\sqrt{\delta}+\sqrt{(n-3)(2 m-\Delta-\delta)+(n-2)\left(\frac{\operatorname{det} D}{\Delta \delta}\right)^{1 /(n-2)}} .
$$

From the above and (4) we arrive at (11).

Equality in (12) holds if and only if $d_{2}=d_{3}=\cdots=d_{n-1}$. Since $G$ has no isolated vertices, equality in (4) holds if and only if $G \cong \frac{n}{2} K_{2}$, for even $n$. This implies that equality in (11) holds if and only if $G \cong \frac{n}{2} K_{2}$, for even $n$.

Remark. Since

$$
(n-2)\left(\frac{\operatorname{det} D}{\Delta \delta}\right)^{1 /(n-2)} \leq \sum_{i=2}^{n-1} d_{i}=2 m-\Delta-\delta,
$$

we have that

$$
\begin{aligned}
& (n-3)(2 m-\Delta-\delta)+(n-2)\left(\frac{\operatorname{det} D}{\Delta \delta}\right)^{1 /(n-2)} \leq \\
\leq & (n-3)(2 m-\Delta-\delta)+2 m-\Delta-\delta=(n-2)(2 m-\Delta-\delta) .
\end{aligned}
$$


Therefore

$$
\begin{aligned}
E(G) & \leq \sqrt{\Delta}+\sqrt{\delta}+\sqrt{(n-3)(2 m-\Delta-\delta)+(n-2)\left(\frac{\operatorname{det} D}{\Delta \delta}\right)^{1 /(n-2)}} \leq \\
& \leq \sqrt{\Delta}+\sqrt{\delta}+\sqrt{(n-2)(2 m-\Delta-\delta)}
\end{aligned}
$$

which means that inequality (11) is stronger than (3).

Proofs of the next theorems are similar to that of Theorem 4, thus omitted.

Theorem 5. Let $G$ be a graph of order $n \geq 2$ and size $m$ without isolated vertices. Then we have

$$
E(G) \leq \sqrt{\Delta}+\sqrt{(n-2)(2 m-\Delta)+(n-1)\left(\frac{\operatorname{det} D}{\Delta}\right)^{1 /(n-1)}}
$$

Equality holds if and only if $G \cong \frac{n}{2} K_{2}$, for even $n$.

Remark. Since

$$
(n-1)\left(\frac{\operatorname{det} D}{\Delta}\right)^{1 /(n-1)} \leq \sum_{i=2}^{n} d_{i}=2 m-\Delta
$$

we have that the following inequality is valid

$$
\begin{aligned}
(n-2)(2 m-\Delta)+(n-1)\left(\frac{\operatorname{det} D}{\Delta}\right)^{\frac{1}{n-1}} & \leq(n-2)(2 m-\Delta)+2 m-\Delta= \\
& =(n-1)(2 m-\Delta) .
\end{aligned}
$$

Therefore

$$
\begin{aligned}
E(G) & \leq \sqrt{\Delta}+\sqrt{(n-2)(2 m-\Delta)+(n-1)\left(\frac{\operatorname{det} D}{\Delta}\right)^{1 /(n-1)}} \leq \\
& \leq \sqrt{\Delta}+\sqrt{(n-1)(2 m-\Delta)}
\end{aligned}
$$

which means that inequality (13) is stronger than (2).

Theorem 6. Let $G$ be a graph of order $n \geq 2$ and size $m$ without isolated 
vertices. Then we have

$$
E(G) \leq \sqrt{2 m(n-1)+n(\operatorname{det} D)^{1 / n}} .
$$

Equality holds if and only if $G \cong \frac{n}{2} K_{2}$, for even $n$.

Remark. Since

$$
n(\operatorname{det} D)^{1 / n} \leq \sum_{i=1}^{n} d_{i}=2 m
$$

we have that

$$
2 m(n-1)+n(\operatorname{det} D)^{1 / n} \leq 2 m(n-1)+2 m=2 m n .
$$

Therefore

$$
E(G) \leq \sqrt{2 m(n-1)+n(\operatorname{det} D)^{1 / n}} \leq \sqrt{2 m n},
$$

which means that inequality (14) is stronger than (1).

Theorem 7. Let $G$ be a graph of order $n \geq 3$ and size $m$ without isolated vertices. Then for any real $\beta$ we have that

$$
E(G) \leq \sqrt{\Delta}+\sqrt{\delta}+\sqrt{\left({ }^{0} R_{1-\beta}(G)-\Delta^{1-\beta}-\delta^{1-\beta}\right)\left({ }^{0} R_{\beta}(G)-\Delta^{\beta}-\delta^{\beta}\right)} .
$$

Equality holds if and only if $G \cong \frac{n}{2} K_{2}$, for even $n$.

Proof. In [22] it was proven that for any real $\alpha$ and $\beta$ holds

$$
{ }^{0} R_{\alpha}(G) \leq \Delta^{\alpha}+\delta^{\alpha}+\sqrt{\left({ }^{0} R_{2 \alpha-\beta}(G)-\Delta^{2 \alpha-\beta}-\delta^{2 \alpha-\beta}\right)\left({ }^{0} R_{\beta}(G)-\Delta^{\beta}-\delta^{\beta}\right)} .
$$

For $\alpha=\frac{1}{2}$, the above inequality becomes

$$
\sum_{i=1}^{n} \sqrt{d_{i}} \leq \sqrt{\Delta}+\sqrt{\delta}+\sqrt{\left({ }^{0} R_{1-\beta}(G)-\Delta^{1-\beta}-\delta^{1-\beta}\right)\left({ }^{0} R_{\beta}(G)-\Delta^{\beta}-\delta^{\beta}\right)} .
$$

Now, from the above and inequality (4) we obtain (15).

Equality in (16) holds if and only if $\beta=\frac{1}{2}$, or $d_{2}=\cdots=d_{n-1}$. Since $G$ has no isolated vertices, equality in (4) holds if and only if $G \cong \frac{n}{2} K_{2}$, for even $n$, which implies that equality in (15) holds under the same condition. 
Remark. For $\beta=1$, the inequality (15) becomes

$$
E(G) \leq \sqrt{\Delta}+\sqrt{\delta}+\sqrt{(n-2)(2 m-\Delta-\delta)},
$$

that is, we obtain (3). This means that (3) is corollary of (15).

By a similar procedure the next theorem can be proved.

Theorem 8. Let $G$ be a graph of order $n \geq 2$ and size $m$ without isolated vertices. Then for any real $\beta$ we have that

$$
E(G) \leq \sqrt{\Delta}+\sqrt{\left({ }^{0} R_{1-\beta}(G)-\Delta^{1-\beta}\right)\left({ }^{0} R_{\beta}(G)-\Delta^{\beta}\right)} .
$$

Equality holds if and only if $G \cong \frac{n}{2} K_{2}$, for even $n$.

Remark. For $\beta=1$, the inequality (17) becomes

$$
E(G) \leq \sqrt{\Delta}+\sqrt{(n-1)(2 m-\Delta)} .
$$

Namely, the inequality (2) is obtained. This means that inequality (2) is a corollary of (17).

Theorem 9. Let $G$ be a graph of order $n$ and size $m$ without isolated vertices. Then for any real $\beta$ we have that

$$
E(G) \leq \sqrt{{ }^{0} R_{1-\beta}(G){ }^{0} R_{\beta}(G)} .
$$

Equality holds if and only if $G \cong \frac{n}{2} K_{2}$, for even $n$.

Remark. For $\beta=1$, the inequality (18) becomes

$$
E(G) \leq \sqrt{2 m n}
$$

that is, the inequality (1) is obtained. This means that inequality (1) is a corollary of (18).

Remark. The inequalities (15), (17) and (18) depend on parameter $\beta$ which is an arbitrary real number. This can be used to obtain a relationship between $E(G)$ and some other graph invariants. For the sake of illustration 
we take $\beta=2$ and from (15), (17) and (18), respectively, we obtain

$$
\begin{aligned}
& E(G) \leq \sqrt{\Delta}+\sqrt{\delta}+\sqrt{\left(I D(G)-\frac{1}{\Delta}-\frac{1}{\delta}\right)\left(M_{1}(G)-\Delta^{2}-\delta^{2}\right)}, \\
& E(G) \leq \sqrt{\Delta}+\sqrt{\left(I D(G)-\frac{1}{\Delta}\right)\left(M_{1}(G)-\Delta^{2}\right)}, \\
& E(G) \leq \sqrt{I D(G) M_{1}(G)} .
\end{aligned}
$$

These inequalities were proven in [4].

Remark. The sum-connectivity index [28] is defined as

$$
S C(G)=\sum_{i \sim j} \frac{1}{\sqrt{d_{i}+d_{j}}},
$$

where summation is performed over all adjacent vertices $v_{i}$ and $v_{j}$ in $G$.

In [6] it was proven that

$$
2 \sqrt{2} S C(G) \leq \sum_{i=1}^{n} \sqrt{d_{i}} .
$$

It can be concluded that all upper bounds obtained for $E(G)$ from (4) are also an upper bounds of $2 \sqrt{2} S C(G)$. Thus, for example, according to the inequalities proved in the present paper, we have that for any real $\beta$ hold

$$
\begin{aligned}
& 2 \sqrt{2} S C(G) \leq \sqrt{\Delta}+\sqrt{\delta}+\sqrt{\left({ }^{0} R_{1-\beta}(G)-\Delta^{1-\beta}-\delta^{1-\beta}\right)\left({ }^{0} R_{\beta}(G)-\Delta^{\beta}-\delta^{\beta}\right)} \\
& 2 \sqrt{2} S C(G) \leq \sqrt{\Delta}+\sqrt{\left({ }^{0} R_{1-\beta}(G)-\Delta^{1-\beta}\right)\left({ }^{0} R_{\beta}(G)-\Delta^{\beta}\right)} \\
& 2 \sqrt{2} S C(G) \leq \sqrt{{ }^{0} R_{1-\beta}(G){ }^{0} R_{\beta}(G)} .
\end{aligned}
$$

When $\beta=1$, we have that

$$
\begin{aligned}
& 2 \sqrt{2} S C(G) \leq \sqrt{2 m n} \\
& 2 \sqrt{2} S C(G) \leq \sqrt{\Delta}+\sqrt{(n-1)(2 m-\Delta)} \\
& 2 \sqrt{2} S C(G) \leq \sqrt{\Delta}+\sqrt{\delta}+\sqrt{(n-2)(2 m-\Delta-\delta)} .
\end{aligned}
$$

The inequality (19) was proven in [28], whereas (20) in [6]. 
Theorem 10. Let $G$ be a graph of order $n \geq 3$ and size $m$ without isolated vertices. Then, for any real $\beta \geq 1$, we have that

$$
E(G) \leq \sqrt{\Delta}+\sqrt{\delta}+(n-2)^{\frac{\beta-1}{\beta}}\left({ }^{0} R_{\frac{\beta}{2}}(G)-\Delta^{\frac{\beta}{2}}-\delta^{\frac{\beta}{2}}\right)^{\frac{1}{\beta}}
$$

Equality holds if and only if $G \cong \frac{n}{2} K_{2}$, for even $n$.

Proof. The inequality (6) can be considered in the following form

$$
\left(\sum_{i=2}^{n-1} p_{i}\right)^{r-1} \sum_{i=2}^{n-1} p_{i} a_{i}^{r} \geq\left(\sum_{i=2}^{n-1} p_{i} a_{i}\right)^{r} .
$$

For $r=\beta, \beta \geq 1, p_{i}=1, a_{i}=\sqrt{d_{i}}, i=2, \ldots, n-1$, the above inequality becomes

$$
\left(\sum_{i=2}^{n-1} 1\right)^{\beta-1} \sum_{i=2}^{n-1} d_{i}^{\frac{\beta}{2}} \geq\left(\sum_{i=2}^{n-1} \sqrt{d_{i}}\right)^{\beta}
$$

that is

$$
\begin{aligned}
& \left(\sum_{i=1}^{n} \sqrt{d_{i}}-\sqrt{\Delta}-\sqrt{\delta}\right)^{\beta} \leq(n-2)^{\beta-1}\left(\sum_{i=1}^{n} d_{i}^{\frac{\beta}{2}}-\Delta^{\frac{\beta}{2}}-\delta^{\frac{\beta}{2}}\right), \\
& \sum_{i=1}^{n} \sqrt{d_{i}} \leq \sqrt{\Delta}+\sqrt{\delta}+(n-2)^{\frac{\beta-1}{\beta}}\left({ }^{0} R_{\frac{\beta}{2}}(G)-\Delta^{\frac{\beta}{2}}-\delta^{\frac{\beta}{2}}\right)^{\frac{1}{\beta}} .
\end{aligned}
$$

Now, from the above and inequality (4) we arrive at (21).

Equality in (22) holds if and only if $d_{2}=d_{3}=\cdots=d_{n-1}$. Equality in (4) holds if and only if $G \cong \overline{K_{n}}$ or $G \cong t K_{2}+(n-2 t) K_{1}, 1 \leq t \leq\left[\frac{n}{2}\right]$. Since $G$ has no isolated vertices, equality in (21) holds if and only if $G \cong \frac{n}{2} K_{2}$, for even $n$.

Remark. For $\beta=1$, from (21) we obtain (4), whereas for $\beta=2$ the inequality ( 3 ) is obtained. For $\beta=4$ and $\beta=6$, respectively, the following inequalities are obtained

$$
\begin{aligned}
& E(G) \leq \sqrt{\Delta}+\sqrt{\delta}+(n-2)^{\frac{3}{4}}\left(M_{1}(G)-\Delta^{2}-\delta^{2}\right)^{\frac{1}{4}} \\
& E(G) \leq \sqrt{\Delta}+\sqrt{\delta}+(n-2)^{\frac{5}{6}}\left(F(G)-\Delta^{3}-\delta^{3}\right)^{\frac{1}{6}}
\end{aligned}
$$


Proofs of the next two theorems are similar to that of Theorem 10, hence omitted.

Theorem 11. Let $G$ be a graph of order $n \geq 2$ and size $m$ without isolated vertices. Then, for any real $\beta \geq 1$, we have that

$$
E(G) \leq \sqrt{\Delta}+(n-1)^{\frac{\beta-1}{\beta}}\left({ }^{0} R_{\frac{\beta}{2}}(G)-\Delta^{\frac{\beta}{2}}\right)^{\frac{1}{\beta}} .
$$

Equality holds if and only if $G \cong \frac{n}{2} K_{2}$, for even $n$.

Remark. For $\beta=4$ and $\beta=6$, respectively, from (23) the following inequalities are obtained

$$
\begin{aligned}
& E(G) \leq \sqrt{\Delta}+(n-1)^{\frac{3}{4}}\left(M_{1}(G)-\Delta^{2}\right)^{\frac{1}{4}} \\
& E(G) \leq \sqrt{\Delta}+(n-1)^{\frac{5}{6}}\left(F(G)-\Delta^{3}\right)^{\frac{1}{6}} .
\end{aligned}
$$

Theorem 12. Let $G$ be a graph of order $n \geq 2$ and size $m$ without isolated vertices. Then, for any real $\beta \geq 1$, we have that

$$
E(G) \leq n^{\frac{\beta-1}{\beta}}{ }^{0} R_{\frac{\beta}{2}}(G)^{\frac{1}{\beta}} .
$$

Equality holds if and only if $G \cong \frac{n}{2} K_{2}$, for even $n$.

Remark. For $\beta=2$ from (24) the inequality (1) is obtained. For $\beta=4$ and $\beta=6$, respectively, the following inequalities are obtained

$$
\begin{aligned}
& E(G) \leq \sqrt[4]{n^{3} M_{1}(G)} \\
& E(G) \leq \sqrt[6]{n^{5} F(G)}
\end{aligned}
$$

Acknowledgment: This paper was partly supported by the Serbian Ministry of Education, Science and Technological Development.

\section{References}

[1] N. Alawah, N. J. Rad, A. Jahanbani, H. Kawarulhaili, New upper bounds on the energy of a graph, MATCH Commun. Math. Comput. Chem. 79 (2018) 287-301. 
[2] A. Ali, L. Zhong, I. Gutman, Harmonic index and its generalizations: extremal results and bounds, MATCH Commun. Math. Comput. Chem. 81 (2009) 249-311.

[3] A. Ali, I. Gutman, E. Milovanović, I. Milovanović, Sum of powers of the degrees of graphs: extremal results and bounds, MATCH Commun. Math. Comput. Chem. 80 (2018) 5-84.

[4] Ş. B. Bozkurt Altındağ, M. Matejić, I. Milovanović, E. Milovanović, Some new upper bounds for the energy of graphs, Sci. Publ. State Univ. Novi Pazar, Ser. A: Appl. Math. Inform. Mech. 14 (2022) 4552 .

[5] K. C. Das, S. A. Mojallal, I. Gutman, Relations between degrees, conjugate degrees and graph energies, Lin. Algebra Appl. 515 (2017) $24-37$.

[6] K. Ch. Das, M. Dehmer, Comparison between the zeroth-order Randić index and the sum-connectivity index, Appl. Math. Comput. 274 (2016) 585-589.

[7] S. Fajtlowicz, On conjectures of Graffiti - II, Congr. Numer. 60 (1987) 187-197.

[8] S. Filipovski, Relations between the energy of graphs and other graph parameters, MATCH Commun. Math. Comput. Chem. 87 (2022) 661672 .

[9] B. Furtula, I. Gutman, A forgotten topological index, J. Math. Chem. 53 (2015) 1184-1190.

[10] I. Gutman, The energy of a graph, Ber. Math.-Statist. Sekt. Forschungsz. Graz 103 (1978) 1-22.

[11] I. Gutman, X. Li (Eds.), Energies of graphs - Theory and Applications, Univ. Kragujevac, Kragujevac, 2016.

[12] I. Gutman, N. Trinajstić, Graph theory and molecular orbitals. Total $\pi$-electron energy of alternant hydrocarbons, Chem. Phys. Lett. 17 (1972) 535-538.

[13] Y. Hu, X. Li, Y. Shi, T. Xu, I. Gutman, On molecular graphs with smallest and greatest zeroth-order general Randić index, MATCH Commun. Math. Comput. Chem. 54 (2005) 425-434.

[14] L. B. Kier, L. H. Hall, Molecular Connectivity in Chemistry and Drug Research, Academic Press, New York, 1976. 
[15] H. Kober, On the arithmetic and geometric means and on Hölder's inequality, Proc. Am. Math. Soc. 9 (1958) 452-459.

[16] X. Li, Y. Shi, I. Gutman, Graph Energy, Springer, New York, 2012.

[17] X. Li, J. Zheng, A unified approach to the extremal trees for different indices, MATCH Commun. Math. Comput. Chem. 54 (2005) 195-208.

[18] M. M. Matejić, P. D. Milošević, E. I. Milovanović, I. Ž. Milovanović, Remarks on general zeroth-order Randić and general sum-conectivity indices, Sci. Publ. State Univ. Novi Pazar, Ser. A: Appl. Math. Inform. Mech. 11 (2019) 11-20.

[19] B. J. McClelland, Properties of the latent roots of a matrix: The estimation of $\pi$-electron energies, J. Chem. Phys. 54 (1971) 640-643.

[20] A. Milićević, S. Nikolić, On variable Zagreb indices, Croat. Chem. Acta 77 (2004) 97-101.

[21] I. Milovanović, E. Milovanović, I. Gutman, Upper bounds for some graph energies, Appl. Math. Comput. 289 (2016) 435-443.

[22] P. Milošević, I. Milovanović, E. Milovanović, M. Matejić, Some inequalities for general zeroth-order Randić index, Filomat 33 (2019) $5249-5258$.

[23] D. S. Mitrinović, P. M. Vasić, Analytic Inequalities, Springer-Verlag, Berlin, 1970.

[24] D. S. Mitrinović, J. E. Pečarić, A. M. Fink, Classical and New Inequalities in Analysis, Kluwer, Dordrecht, 1993.

[25] V. Nikiforov, The energy of graphs and matrices, J. Math. Anal. Appl. 326 (2007) 1472-1475

[26] V. Nikiforov, Extreme of graph energies, Lin. Algebra Appl. 482 (2015) 158-190.

[27] S. Nikolić, G. Kovačević, A. Milićević, N. Trinajstić, The Zagreb indices 30 years after, Croat. Chem. Acta 76 (2003) 113-124.

[28] B. Zhou, N. Trinajstić, On novel connectivity index, J. Math. Chem. 46 (2009) 1252-1270. 